\title{
Soccer (football) ocular injuries: An important eye health problem
}

\section{J A Capão Filipe}

Laboratory experiments have confirmed the mechanism of soccer ball eye injury and the feasibility of a protective eyewear in soccer

T he ideal scenario for a sports (or any other) eye injury is for it never to have happened. According to the US Centers for Disease Control and Prevention, "injury is probably the most unrecognized major health problem facing the nation today, and the study of injury presents unparalleled opportunities for reducing morbidity and mortality and for realizing significant savings in both financial and human terms-all in return for a relatively modest investment." ${ }^{\prime 1}$ The social cost of eye trauma, the most common ophthalmic indication for hospitalisation, is enormous. National projections estimate annual US hospital charges of $\$ 175$ million to \$200 million for 227000 eye trauma hospital days. ${ }^{2}$ Tan, in a recent $B J O$ editorial, commented on prevention of blindness programmes, and stated that "let us then remind ourselves of prevention of blindness programmes which have the potential to do the greatest good for the most people." ${ }^{\prime 3}$ By carefully evaluating the underlying mechanisms, patterns, and rates of injury in a given sport, it is possible to design and implement extremely effective preventive programmes.

Soccer ocular injury is an important eye health problem in Europe and probably worldwide. ${ }^{4-18}$ In these series, contrary to previous ophthalmology teaching that eye injuries are rarely caused by balls larger than 4 inches in diameter, the large diameter soccer ball was responsible for most soccer injuries. In 1994, Vinger focused on some pertinent questions related to soccer eye injuries." "How is sufficient energy transmitted from the large ball to the eye to result in severe lesions in many injured players? Are the eye injuries caused by ball deformation? Does underinflation result in more eye injuries than properly inflated balls? Are sports eye protectors currently in use for racket sports sufficient to prevent injury?" In the same chapter he proposed the future method of investigation, and stated that "impact testing of balls at varying speeds and pressures, with and without various eye protectors in place, using high-speed photography, should, in combination with prospective over the next several years." In fact, several years elapsed to the publication, in this issue of the BJO (p 167), of a paper about the mechanism of soccer eye injuries, where the authors tried to address some of above mentioned questions and to lead off its prevention.

Although the differences between the opening of the bony orbit $(1.4 \times 1.6$ inches $)$ and the diameter of a standard soccer ball (8.6 inches), the laboratory experiments carried out confirmed that soccer balls deform significantly on impact, allowing a small "knuckle" of the ball to enter the orbit and impact the globe. Still more, it was proved that the soccer ball is unique among the sports balls tested: orbital penetration is lower, but the time in the orbit is longer, and during rebound a secondary suction effect is produced on the orbital contents. The expansion of the eyeball perpendicular to the direction of impact has been proposed as the major cause of the contusion injuries. ${ }^{20}$ The suction component most likely adds to the distortion of the globe anatomy, which can explain the findings of the

\section{Key messages}

- The soccer ball is unique among the sports balls tested: orbital penetration is lower, but the time in the orbit is longer and, during rebound, a secondary suction effect is produced on the orbital contents.

- We strongly recommend that soccer protective eyewear conforming to ASTM F803 should be worn.

- The future of soccer ocular injuries prevention has already started! injury studies, answer these questions

\section{MECHANISM OF INJURY}

clinical studies, showing that soccer injuries were disproportionately severe. $^{67912}$ On the other hand, the experiments showed that soccer ball penetration is not significantly related to the size of the ball or to the pressure, and even when they are not underinflated, they can deform on impact. These results support, in part, the most important finding in our previous prospective study that soccer eye injuries affected young athletes independently of age, sex and type of soccer, level of expertise or player position. ${ }^{7}$

The assumption that when a large object such as a soccer ball hits the eye, more energy is directly transmitted to the exposed temporal retina while the nasal retina is protected by the nose, could explain the predilection of soccer eye injury lesions to the superotemporal quadrant found in our studies. Therefore, we should not forget that it is essential to examine the peripheral retina of all eyes that have had a soccer ball contusion injury, with particular attention for that quadrant.

\section{SOCCER EYE PROTECTOR}

If protective devices are necessary then performance standards must be written to ensure that the protective devices will meet the visual requirements of the game while reducing the probability of injury to a specified level. The majority of sports eyewear standards written in the United States comes under the jurisdiction of ASTM (American Society for Testing and Materials, http://www.astm.org/), a non-profit corporation organised, as early as 1898, for development of voluntary standards arrived at by consensus, with strict guidelines for due process, among all interested parties. ${ }^{21}{ }^{22}$ With the publication of this article we have the valuable information that protectors that comply with the requirements of ASTM standard F803 (and have polycarbonate lenses) will prevent eye contact with a soccer ball.

The need for protective eyewear in soccer remained far less clear than for other sports. Their use by children or adolescents presenting underdeveloped orbital structures was already previously considered. Now, we strongly recommend that soccer protective eyewear conforming to ASTM F803 should be worn also by adults, particularly for subjects who require prescription lenses, for functionally one eyed athletes, and for those who have had refractive surgical procedures that weaken the eye. The use of protective eyewear in soccer was recently popularised by a well known professional player of the Dutch national team, Edgar Davids. This fact led a sports equipment company to 
develop soccer eyewear. The future of soccer ocular injuries prevention has already started!

\section{CONCLUSION}

In this new millennium, soccer may become the most common cause of sports eye injury worldwide. As physicians and surgeons, our mindset is towards the evaluation and treatment of eye disease. Injuries are predictable and, for most of them, preventable if we all make an eye safety prescription part of our routine.

Standards are designed to be revised as experience is gained. No matter how well the protector performs on paper or in the testing laboratory, it is the use by thousands of players and continued injuries monitoring that prove the protective value or demonstrate the failures of a particular design. Since 1992 we implemented a sports ophthalmology unit in our department of ophthalmology to follow athletes with sports related ocular lesions. The observation and treatment of these patients by specially trained personnel, using standardised protocols, will allow a better care, the accumulation of important epidemiological data, and it may have a special educational role for preventive measures. Alternatively, the development of further laboratory experiments combined with a better understanding of the biomechanics of eye injury will reveal additional alterations as a consequence of soccer ball blunt injury. International and national governing bodies, such as the "Federation Internationale de Football Association" (FIFA), should also be involved in these discussions and deliberations.

In the final comment of this editorial we have to honour Dr Paul Vinger for all the work developed in sports ophthalmology. Dr Vinger has devoted years of research time to the study of eye injuries, their mechanism, and prevention. These studies have led to the establishment of standards of eye protective equipment in racket sports, ski goggles, hockey face helmets, baseball face protectors, fencing headgear, equestrian head protection, paintball, and, since this issue of $B J O$, soccer eye protection. Br J Ophthalmol 2004;88:159-160. doi: 10.1136/bjo.2003.031518

Correspondence to: J A Capão Filipe, Sports Ophthalmology Unit, Department of

Ophthalmology, University of Porto School of Medicine, S João Hospital, 4200-319 Porto, Portugal; jacapaofilipe@netcabo.pt

\section{REFERENCES}

1 Viano DC. A blueprint for injury control in the United States. Public Health Rep 1990;105:329-33.

2 Tielsch JM, Parver LM. Determinants of hospital charges and length of stay for ocular trauma. Ophthalmology 1990;97:231-7.

3 Tan D. Who's afraid of prevention of blindness? Br J Ophthalmol 2000;84:943-4.

4 Barr A, Baines PS, Desai P, et al. Ocular sports injuries: the current picture. Br $J$ Sports Med 2000;34:456-8

5 Burke MJ, Sanitato JJ, Vinger PF, et al. Soccerball-induced eye injuries. JAMA 1983;249:2682-5.
6 Capão-Filipe JA, Castro-Correia J. [Sportsrelated ocular injuries in Portugal]. Rev Port Med Desp 1993;11:65-76.

7 Capão Filipe JA, Fernandes VL, Barros $\mathrm{H}$, et al. Soccer-related ocular injuries. Arch Ophthalmol 2003; 121:687-94

8 Drolsum L. Eye injuries in sports. Scand J Med Sci Sports 1999:9:53-6.

9 Filipe JAC, Barros H, Castro-Correia J. Sportsrelated ocular injuries in Portugal. A three year follow-up study. Ophthalmology 1997;104:313-8.

10 Gregory PTS. Sussex eye hospital sports injuries. $\mathrm{Br} J$ Ophthalmol 1986:70:748-50.

11 Horn EP, McDonald HR, Johnson RN, et al. Soccer ball-related retinal injuries: a report of 13 cases. Retina 2000;20:604-9.

12 Larrison WI, Hersh PS, Kunzweiler T, et al. Sports-related ocular trauma. Ophthalmology 1990;97:1265-9.

13 Larrison WI. Golf and soccer. In: Zagelbaum BM, ed. Sports ophthalmology. Cambridge, USA Blackwell Science, 1996:125-31.

14 MacEwen CJ. Sport associated eye injury: a casualty department survey. $\mathrm{Br} J$ Ophthalmol 1987:71:701-5.

15 MacEwen CJ. Eye injuries: a prospective survey of 5671 cases. Br J Ophthalmol 1989;73:888-94

16 Pikkel J, Gelfand Y, Miller B. [Incidence of sportsrelated eye injuries]. Harefuah 1995: 129:249-50.

17 Verdaguer TJ. Juvenile retinal detachment Am J Ophthalmol 1982;93:145-56.

18 Vinger PF. Sports-related eye injury. A preventable problem. Surv Ophthalmo 1980;25:47-51.

19 Vinger PF. The eye and sports medicine. In: Tasman W, Jaeger EA, eds. Duane's clinical ophthalmology. Philadelphia: JB Lippincott, 1994:5:45.

20 Schepens CL. Contusion Trauma. In: Schepens CL, ed. Retinal detachment and allied diseases. Philadelphia: WB Saunders, 1983:1:71-84.

21 Vinger P. Eye safety testing and standards. Ophthalmol Clin N Am 1999;12:345-58. 22 Hulse W. Sports equipment standards. In: Vinger $\mathrm{P}$, Hoerner $\mathrm{E}$, eds. Sports injuries: the unthwarted epidemic. Littleton, MA: Publishing Sciences Group, 1981:378-82.

Ho! hi!

Oh, my eye!

My mind may be wandering, but I

confess

I believe it is Old Deuteronomy!"

From TS Eliot's: Old Possum's Book of Practical Cats

\section{W V Good}

\section{Each time it appears to have been eliminated, it resurfaces}

\author{
Old Deuteronomy's lived a long \\ time; \\ He's a Cat who has lived many lives \\ in succession. \\ He was famous in proverb and \\ famous in rhyme \\ A long while before Queen \\ Victoria's accession. \\ Old Deuteronomy's buried nine \\ wives \\ And more-I am tempted to say, \\ ninety-nine;
}

\author{
And his numerous progeny prospers \\ and thrives \\ And the village is proud of him in his \\ decline. \\ At the sight of that placid and bland \\ physiognomy, \\ When he sits in the sun on the \\ vicarage wall, \\ The Oldest Inhabitant croaks: \\ "Well, of all ... \\ Things. . . Can it be . . really! . . . \\ No!. . . Yes!. .
}

ife is bracketed by two turbulent periods: birth and death. At both -ends, the medical field struggles to expand the lifespan, to promote viability in increasingly premature infants and increasingly aged populations. At the limits of viability there is a price to pay to sustain life. Statistics on viability in preterm infants show that many more low birthweight infants survive, but with substantial morbidity. ${ }^{1}$ The same is true at the other end of the life spectrum. Most medical costs and morbidities occur during the final days and weeks of life.

In the United States, the premature birth rate for infants born at less than 37 weeks gestational age has increased in the past several years. ${ }^{2}$ Smaller 
infants who previously would have succumbed to prematurity now survive. Morbidity has not declined. Neurodevelopmental damage, chronic lung disease, liver and heart disease remain important problems. Recent evidence suggests that many low birthweight infants suffer CNS damage, at least when brain volume is assessed. ${ }^{3}$ Despite wonderful advances in our ability to sustain life, too many premature infants graduate from the nursery with significant and often permanent medical problems.

In this issue of the BJO ( $\mathrm{p}$ 239) Allegaert and colleagues from the EpiBel Study Group give us some insight into the risk of severe retinopathy of prematurity (ROP) in the youngest of survivors-those born at gestational ages $\leqslant 26$ weeks. A lively debate has erupted over the past decade as to whether ROP remains a significant healthcare problem, at least in western countries. Some studies suggest that the incidence of ROP has been reduced, ${ }^{4}$ but not all agree that this is the case. ${ }^{5}$ The argument goes something like this. Children born 15 years ago were managed differently and therefore cannot rightly be compared to current day infants. Neonatal advances may have reduced the incidence of ROP. Laser treatment has replaced cryoablation and is more effective. Therefore we don't see as much ROP, and severe cases of ROP are managed in ways that dramatically improve structural and functional outcomes.

The answer to the debate about the significance and modern incidence of ROP will be unknown until larger scale studies are completed, ${ }^{6}$ but the findings from the EpiBel Group suggest that ROP remains an important problem, at least in the youngest gestational age infants. The EpiBel study found threshold ROP in $19.8 \%$ of infants born between 22 and 26 weeks gestational age, and severe ROP ( $\geqslant$ stage 3 ) in $25.5 \%$. It is difficult to compare these findings to other studies, since other studies have used different criteria for study entry (for example, $\leqslant 1250 \mathrm{~g}$ ), but the risk of severe ROP in the EpiBel Study is high by any standard. The risk is particularly high at the limit of viability. Ineluctably, ROP will remain a significant problem as smaller infants survive, because ROP is a disease of the smallest and youngest; those on the edge of life.

For argument's sake, taking the position that ROP is curable with laser or cryotherapy, there might be no reason for concern, despite the findings reported in this issue. This argument ignores the important findings from the CRYO-ROP study, that ocular morbidity is significant in infants with threshold ROP. Visual acuity is frequently worse than $6 / 12$ in infants with threshold $\mathrm{ROP}^{7}$ and myopia, amblyopia, and strabismus are common. Also overlooked in this debate is the risk of cerebral (cortical) visual impairment, a problem which also often resides on the margins of viability.

This report from the EpiBel Group demonstrates a new finding, that renal insufficiency is associated with ROP. While the significance of this finding is unclear, it demonstrates again that the smallest and sickest infants are most likely to develop significant ROP. Another intriguing finding is the association between CRIB and Apgar scores and significant ROP. These scores could identify infants at risk for ophthalmic morbidity, although a prospective study of this issue seems advisable before drawing this conclusion. To the extent that Apgar and CRIB scores can be modified, the incidence and severity of ROP and other diseases of preterm infants might be reduced. On the other hand, these scores could simply be markers for the sickest, smallest, and youngest infants.

The authors also remind us that infants with severe ROP usually sustain damage to other organ systems. Threshold ROP was the sole marker for morbidity at discharge in only $8 \%$ of infants in this study. This finding is familiar to paediatricians and ophthalmologists who care for children with advanced ROP. These children and their caregivers face complicated challenges caused by ophthalmic and neurological morbidity.

Is ROP a disease of the past? As long as infants survive on the margins of viability it is not. ROP has been declared dead on many previous occasions, only to be resurrected as viability at the margins of life improves. ROP is like the cat with nine lives. Each time it appears to have been eliminated, it resurfaces. We should thank Allegaert and colleagues for their important report, which reminds us of the limitations we face as physicians involved in the care of the youngest, most fragile, infants.

Br J Ophthalmol 2004;88:160-161. doi: 10.1136/bjo.2003.031575

Correspondence to: W V Good, SmithKettlewell Eye Research Institute, 2318 Fillmore Street, San Francisco, CA 94115, USA; Good@Ski.org

\section{REFERENCES}

1 Harper RG, Rehman KU, Sia C, et al. Neonatal outcome of infants born at 500 to 800 grams from 1990 through 1998 in a tertiary care center. J Perinatol 2002;22:555-62.

2 Goldenberg RL, Jobe AH. Prospects for research in reproductive health and birth outcomes. JAMA 2001;285:633-9.

3 Peterson BS, Anderson AW, Ehrenkranz R, et al. Regional brain volumes and their later neurodevelopmental correlates in term and preterm infants. Pediatrics 2003;111:939-48.

4 Bullard SR, Donahue SP, Feman SS, et al. The decreasing incidence and severity of retinopathy of prematurity. J AAPOS 1999;3:46-52.

5 Termote J, Schalij-Delfos NE, Donders AR, et al. The incidence of visually impaired children with retinopathy of prematurity and their concomitant disabilities. J AAPOS 2003;7:131-6.

6 Good WV, Hardy RJ. The Multicenter Study of Early Treatment for Retinopathy of Prematurity (ETROP). Ophthalmology

2001;108:1013-14.

7 Multicenter Trial of Cryotherapy for Retinopathy of Prematurity. Ophthalmological outcomes at 10 years. Arch Ophthalmol 2001;119:1110-18.

\section{Update of the vascular model of AMD}

\section{E Friedman}

\section{Are statins or antihypertensives protective?}

$\mathrm{T}$ he observation that age related macular degeneration (AMD) and atherosclerosis share risk factors and pathogenetic mechanisms ${ }^{1}$ has led to the development of a hypothesis that is identified as a haemodynamic ${ }^{2}$ or vascular $^{1}$ model of the pathogenesis of AMD. It holds that AMD is a vascular disorder characterised by impairment of choroidal perfusion of the retinal pigment epithelium (RPE). This model, evolved over four decades, ${ }^{3}$ is now updated to incorporate recently reported evidence ${ }^{4}$ that the changes affecting Bruch's membrane in age and AMD involve lipoproteins processed by the RPE. The model proposes that these lipoproteins accumulate in drusen and in Bruch's membrane because the choriocapillaris does not clear them.

Theoretical models of disease are evaluated by how much they explain and by their success in making useful 
predictions. As the vascular model, by emphasising the pathogenetic importance of atherosclerotic processes and increased intravascular pressure, essentially predicts that statins and antihypertensive agents should have a protective effect on AMD, it is instructive to review the evidence to date.

\section{THE VASCULAR MODEL OF THE PATHOGENESIS OF AMD (FIG 1)}

- This model asserts that choroidal vascular resistance is increased by decreased compliance of ocular tissues, $^{5}$ as a result of progressive infiltration with lipid. It is likely that progressive narrowing of the macular choriocapillaris with age $^{3}$ also contributes to the increased resistance. $^{6}$

- While the systemic circulation is the source of the lipid in the sclera ${ }^{7}$ and choroidal vasculature, there is evidence that the RPE is the source of lipids in drusen and in Bruch's membrane. ${ }^{4}$

- The vascular model contends that impairment of choroidal perfusion, consisting of decreased blood flow and elevated hydrostatic pressure, compromises the processing of outer segment lipid by the RPE and the clearance of the lipoproteins secreted by the RPE. This results in drusen, pigment changes, and geographic atrophy, as well as calcification and fracture of Bruch's membrane. Progressive accumulation of hydrophobic lipids in Bruch's membrane may compromise RPE function further by decreasing its hydraulic conductivity. ${ }^{8}$
- The model proposes that the combination of elevated choriocapillary pressure, breaks in Bruch's membrane, and vascular endothelial growth factor (VEGF) causes choroidal neovascularisation (CNV).

\section{STRENGTHS AND WEAKNESSES OF THE VASCULAR MODEL}

A major strength of the model is that it explains most of what is known about the epidemiology, clinical course, and histopathology of AMD. It explains why atherosclerotic risk factors in general, and systemic hypertension in particular, are risk factors for AMD. It uniquely explains why hyperopia ${ }^{9}$ is a risk factor in AMD, and myopic eyes are relatively protected.

A weakness of the model is that it is not yet technically feasible to confirm the proposed elevated choriocapillary pressure in AMD by direct measurement. Similarly, the stiffness of Bruch's membrane is also not measurable, and the methods available to estimate the coefficient of scleral rigidity are notoriously inaccurate.

Another weakness is that the lower incidence of AMD in African Americans can be explained by the model only by proposing that the compliance of ocular tissues may be greater in African Americans than in white people. That has yet to be determined.

Proponents of competing models assert that choroidal vascular changes in AMD are the result, ${ }^{10}$ rather than the cause, of RPE damage, as proposed by the vascular model. Recent reports, ${ }^{11}$ however, indicate that the fellow eyes of patients with unilateral neovascular AMD are characterised by impaired choroidal perfusion, in the absence of

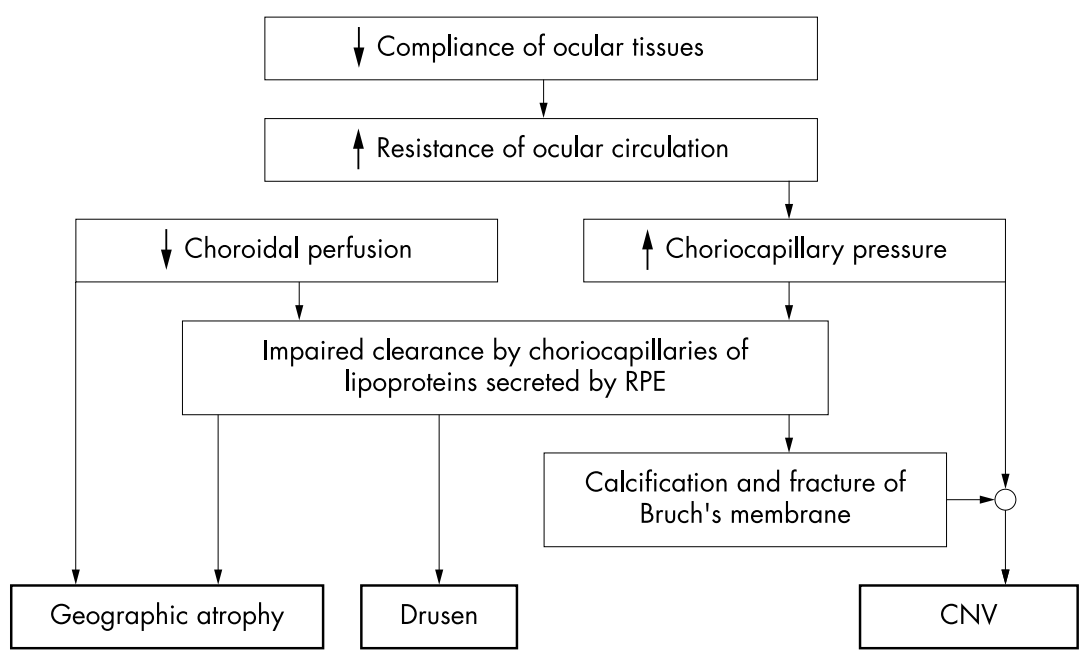

Figure 1 Schematic representation of the vascular model of the pathogenesis of age related macular degeneration. significant RPE damage. This suggests that the choroidal vascular deficit precedes and may cause the RPE changes.

\section{SYSTEMIC HYPERTENSION}

The vascular model proposes that the increase in intravascular pressure results from an increase in the post-capillary resistance of the choroid. Increased resistance was demonstrated by prolonged choroidal filling times ${ }^{12}$ and by colour $^{6}$ and laser Doppler imaging. ${ }^{13}$ The presence of increased intravascular pressure in AMD is suggested by phlebosclerosis of the intrascleral portion of the vortex veins, ${ }^{14}$ distension and tortuosity of the ciliary arteries ${ }^{15}$ and vortex veins, and the perivenular distribution of drusen.?

Systemic hypertension, as well as subclinical atherosclerosis, increases the risk of neovascular and non-neovascular AMD in case-control ${ }^{16}$ as well as population based ${ }^{17}$ epidemiological studies. Systemic hypertension is associated with narrowing of the terminal arteriole, with the ensuing increase in intravascular pressure upstream of the narrowing, largely sparing the capillary bed. The model proposes that when the increased pressure is superimposed on an elevated capillary pressure vascular decompensation results.

\section{ACE INHIBITORS AND AII ANTAGONISTS AND AMD}

While epidiomological studies have established the risk of AMD attributable to systemic hypertension and elevated blood pressure, there are no reports of controlled clinical trials documenting the effectiveness of antihypertensives in lowering the risk of AMD.

Angiotensin converting enzyme (ACE) inhibitors and angiotensin II (AII) antagonists, two classes of drugs that reduce the activity of the renninangiotensin II system, are among the most effective agents in lowering blood pressure, preventing hypertensive end organ damage and mortality, with minimal disturbing side effects. ${ }^{18}$ Laboratory investigations also suggest that they may have possible advantages in controlling blood pressure in AMD. ${ }^{19-21}$

\section{STATINS AND AMD}

Statins reduce serum cholesterol levels by competitively inhibiting an enzyme involved in the synthesis of cholesterol. ${ }^{22}$ They cause significant reduction of cardiovascular and cerebrovascular morbidity and mortality and are useful in the management of rheumatoid arthritis, multiple sclerosis, and Alzheimer's disease. Undesirable side effects of statins are offset by its benefits by a wide margin. 
The first study to suggest a beneficial effect of statins on the course of AMD is a cross sectional survey in Britain, ${ }^{23}$ in which participants taking statins had one seventh the risk of developing AMD, compared to those who did not. However, the odds ratio for macular degeneration was 0.14 with an extremely wide confidence interval (0.02 to 0.83). A small study of elderly Australians $^{24}$ reported a lower rate of progression (3.6\% $v 13 \%$ ) of early AMD in those taking statins at baseline. While the effect was large, it was not statistically significant. Two additional reports $^{25}{ }^{26}$ at the most recent ARVO meeting suggest a beneficial effect of statins on AMD. Based on retrospective reviews of medical records, they both report that progression of AMD was slowed with statin use in a statistically significant manner. Two population based studies ${ }^{27} 28$ report that statins showed no protective effect.

The largest and most convincing study to date ${ }^{29}$ reports that 550 incident cases of early AMD were $70 \%$ less likely to have filled a statin prescription than 5500 matched controls.

The authors of most of these studies emphasise the preliminary nature of the reports and recommend that they be followed by larger clinical trials.

\section{FUTURE STUDIES}

Reports of the protective effects of statins, ACE inhibitors, and AII antagonists on AMD are consistent with, even predicted by, the vascular model. However, even if their effectiveness is confirmed, the mechanisms by which they confer protection may remain uncertain. ${ }^{17}$ The statins can act indirectly by lowering serum cholesterol or directly on the processing of lipids by the RPE. It may not be the lowering of lipid levels by statins nor of blood pressure by hypertensives that offers protection, but rather other properties of these agents. It would be prudent to ascertain whether the preliminary reports of the possible effectiveness of these drugs are confirmed before speculating on the mechanism of their action. Confirmation should take the form of prospective, adequately powered, randomised, controlled clinical trials.

\section{SUMMARY AND CONCLUSIONS}

- The vascular model of AMD is updated. It highlights the roles of the atherosclerotic process and blood pressure in the pathogenesis of the disorder.

- Epidemiological studies suggest that statins and hypertensives may lower the risk of AMD. These reports are sufficiently promising to warrant adequately designed and properly executed clinical trials.

- Confirmation of the protective effect of statins and antihypertensives will lend further support to the vascular model.

\section{ACKNOWLEDGEMENTS}

This work was supported in part by the Solman Friedman Research Fund; The Harold Alfond Research Fund; The Ben Wunsch Research Fund; and The Elinor Bronstein Research Fund, all in Boston, Massachusetts, USA.

Br J Ophthalmol 2004;88:161-163.

doi: 10.1136/bjo.2003.036277

Correspondence to: E Friedman, Department of Ophthalmology, Harvard Medical School, Retina Service, Massachusetts Eye and Ear Infirmary, Boston, and Massachusetts Eye and Ear Infirmary, 243 Charles Street, Boston, MA 02114, USA; ephraimfriedman@mac.com

\section{REFERENCES}

1 Friedman $\mathrm{E}$. The role of the atherosclerotic process in the pathogenesis of age-related macular degeneration. Am J Ophthalmol 2000; 130:658-63.

2 Friedman E. A hemodynamic model of the pathogenesis of age-related macular degeneration. Am J Ophthalmol 1997; 124:677-82.

3 Friedman E, Smith T, Kuwabara T. Senile choroidal vascular patterns and drusen. Arch Ophthalmol 1963;69:220-30.

4 Malek G, Li CM, Guidry C, et al. Apolipoprotein B in cholesterol-containing drusen and basal deposits of human eyes with age-related maculopathy. Am J Pathol 2003; 162:413-25

5 Friedman $E$, lvry $M$, Ebert $E$, et al. Increased scleral rigidity and age-related macular degeneration. Ophthalmology 1989;96:104-8.

6 Friedman E, Krupsky S, Lane A, et al. Ocular blood flow velocity in age-related macular degeneration. Ophthalmology 1995;102:640-6.

7 Broekhuyse RN. The lipid composition of aging sclera and cornea. Ophthalmologica 1975; 171:82-5.

8 Bird A, Marshall J. Retinal pigment epithelial detachments in the elderly. Trans Ophthalmol Soc UK 1986; 105:674-82.

9 Ikram MK, van Leeuwen R, Vingerling JR, et al. Reltionship between refraction and prevalent as well as incident age-related maculopathy: the Rotterdam Study. Invest Ophthalmol Vis Sci 2003;44:3778-82.
10 Del Priore LV, Kaplan H, Hornbeck R, et al. Retinal pigment epithelial debridment as a model for the pathogenesis and management of age related macular degeneration. Am J Ophthalmol 1996:122:629-43.

11 Dimitrova G, Tamaki Y, Kato S. Retrobulbar circulation in patients with age-related maculopathy. Eye 2002;16:580-6.

12 Ciulla TA, Harris A, Kagemann L. Choroidal perfusion perturbations in non-neovascular age related macular degeneration. $\mathrm{Br} J$ Ophthalmo 2002;86:209-13

13 Grunwald JE, Hariprasad SM, DuPont J. Foveolar choroidal blood flow in age-related macular degeneration. Invest Ophthalmol Vis Sci 1998;38:385-91.

14 Friedman E. Central serous choroidopathy: pathogenesis and treatment. In: Brockhurst RI, Boruchoff SA, Hutchinson BT, Lessell S, eds. Controversy in ophthalmology. Philadelphia: Saunders, 1977:706-9.

15 Bischoff P, Flower R. High blood pressure in choroidal arteries as a possible pathogenetic mechanism in senile macular degeneration [letter]. Am J Ophthalmol 1983;96:388-99.

16 Hyman L, Schachat AP, $\mathrm{He} Q$, et al. Hypertension, cardiovascular diseases, and agerelated macular degeneration. Arch Ophthalmol 2000;117:351-8

17 Van Leeuwen R, Ikram MK, Vingerling JR, et al. Blood pressure, atherosclerosis, and the incidence of age-related maculopathy: the Rotterdam Study. Invest Ophthalmol Vis Sci 2003;44:3771-7.

18 Moore MA. Drugs that interrupt the renninangiotensin system should be among the preferred initial drugs to treat hypertension. $J$ Clin Hypert 2003;5:137-44.

19 Sjolie AK, Chaturvedi N. The retinal renninangiotensin system: implications for therapy in diabetic retinopathy. $J$ Hum Hypertens 2002;(Suppl 3):S42-6.

20 Wilson AS, Hobbs BG, Shen WY, et al. Argon laser photocoagulation-induced modification of gene expression in the retina. Invest Ophthalmol Vis Sci 2003;44:1426-34

21 Hamdi HK, Reznik J, Castellon R, et al. Alu DNA polymorphism in ACE gene is protective for agerelated macular degeneration. Biochem Biophys Res Commun 2002;295:668-72.

22 Veillard NR, Mach F. Statins: the new aspirin? CMLS, Cell Mol Life Sci 2002;59:171-6.

23 Hall NF, Gale CR, Syddall H, et al. Risk of macular degeneration in users of statins: cross sectional study. BMJ 2001;323:375-6.

24 McCarty CA, Mukesh BN, Guymer RH, et al. Cholesterol- lowering medications reduce the risk of age-related maculopathy progression. Med J Aust 2001;175:340.

25 Wilson H, Bhatt HRF, Schwartz DM, et al. Statin therapy is associated with decreased odds of developing noevascular age-related macular degeneration. Invest Ophthalmol Vis Sci (Suppl) 2003:1804.

26 Chavis PS, Castillo IG Schwartz SG, et al. Risk factors for age-related macular degeneration. Invest Ophthalmol Vis Sc (Suppl) 2003:5038.

27 Van Leeuwen R, Vingerling JR, Hofman A, et al. Cholesterol lowering drugs and risk of agerelated maculopathy: prospective cohort study with cumulative exposure measurement. $B M$ 2003;326:255-6.

28 Klein R, Klein B, Tomany SC, et al. Relation of statin use to the 5 -year incidence and progression of age-related maculopathy. Arch Ophthalmol 2003; 121:1151-5.

29 McGwin Jr G, Owsley, Curcio CA, et al. The association between statin use and age-related maculopathy. Br J Ophthalmol 2003;87:1121-5. 